\title{
Information about Artificially Intelligent Nanoarray for Detection of Various Diseases in Nanomedicine
}

\author{
V.M. Aroutiounian \\ Yerevan State University, Yerevan, Armenia \\ Email: kisahar@ysu.am
}

(Received: September 12, 2021; September 21, 2021; Accepted: September 27, 2021)

https://doi.org/10.52853/18291171-2021.14.3-148

Physicians evaluated their patients by exhaled volatile organic compounds (VOCs) two and a half millennia. Capabilities of detection of various diseases using the analysis of the breath and how it linked to different diseases discussed were discussed, for example, in $[1,2]$. Data on the surveillance capabilities of diseases in the allocation of specific gases are collected in Table 1 in [1]. Note that the analysis of the breath allows detecting (diagnose) cancer of stomach and ovaries, rheumatoid disease, acute myocardial infarction, gum disease, tooth decay, bacterial imbalance on the tongue, impacted wisdom teeth, dehydration, sleep apnea, gastritis, diabetes and stomach ulcers, as well as many other diseases, can be carried out. Of course, the possibility of physical-chemical studies of gas-phase are not limited to the analysis. Samples of internal abdominal gases can collect and examine from different sections of the bronchi with bronchoscopy, from the stomach with gastroscopy, from the colon with colonoscopy, from the bladder with cystoscopy, from the uterus during hysteroscopy. Today we are talking about the possibility of developing an individual metabolic profile of the patient. The use of semiconductor gas sensors for detecting the concentration of detectable gas released in patients (acetone in diabetes, for example) is widely discussed in the literature [3-5].

Determination of the concentration of chemical compounds in explosives using gas sensors allows the diagnosis of the disease already in the early stages and makes it possible to control treatment. In addition to nitrogen, oxygen, and water vapor, explosives contain various gases in a sick person. By the concentration of acetone and the amount of current or voltage passing through the semiconductor sensor, for example, one can judge the degree of diabetes, acute heart failure, lung cancer; hydrogen peroxide and nitric oxide-asthma and other pulmonary diseases, cancer of the digestive system; ammonia and hydrogen sulfide - hepatitis and cirrhosis; hydrogen and methane, a number of diseases of the digestive system, etc. The current study of head, ovarian, bladder, prostate, kidney, gastric, and neck cancer, Crohn's disease, ulcerative colitis idiopathic and atypical Parkinson's, multiple sclerosis, pulmonary hypertension, and other diseases is started now using nanoarrays and artificial intelligence methods.

Analysis of exhaled breath should be analyzed today using low-cost, low-energy, micro-and nanosized semiconductor gas sensors and based on them matrices which are significantly smaller, easier to use, and less expensive than other sensing devices [6-8]. It was established that such nanosensors or matrices made from them allow detecting (recognition) of VOC or other gases exhaled by [7-9]. Despite advances in the detection of VOCs, we should note that indicating VOCs is promising alone for a relatively narrow spectrum of diseases [7, 8]. An additional limitation is a problem of synthesizing highly selective nanomaterials for each VOC, notably when they are nonpolar [10]. A complementary approach for disease detection and classification diseases relies on cross-reactive semi-selective nanotechnology-based sensor arrays, using pattern recognition [7, 8]. Such approach is referred in as "artificially intelligent nanoarray" [11-14]. It is more suitable for rapid diagnostic methods in which evaluation of a VOC compendium is qualitative and semiquantitative, with selectivity being achieved through pattern recognition of the compendium [7,8]. 
Due to cross-reactivity, each sensor responds to a variety of VOCs, thereby allow sensing and analysis of individual components from multicomponent samples $[11,12,15,16]$. The concept of the artificially intelligent nanoarray is based on the ability of each sensor to detect all or part of the sample compounds. These sensors may have a sensitivity to a specific analyte (or VOC) lower than that of a selective sensor, but they are more versatile in detecting multicomponent and complex VOC mixtures in different atmospheres (including those for which the (nano)arrays were not originally designed) $[11,12,16,17]$.

A multicenter clinical assessment was carried out in [18] to examine the probability of a single artificially intelligent nanoarray to detect and classify a range of disease types to explore the potential of this approach to modern clinical practice. A total of 2808 breath samples were collected from 1404 subjects having one of 17 different disease types that had been put into three main categories (cancerous, inflammatory, and neurological diseases) and one group of uncorrelated diseases, as well as a group of healthy controls. The effects of primary demographic and environmental confounding factors (e.g., sex, age, smoking habits, and geographical location) on the diagnosis and the classification results were examined. All breath samples were examined using gas chromatography linked with mass spectrometry (GC-MS). In contrast to an artificially intelligent nanoarray that analyzes collective breath VOC patterns, GC-MS identifies and quantifies specific components (VOCs).

The analysis was carried out in [18] on breath samples collected in a controlled manner from 1404 eligible subjects collected between January 2011 and June 2014 from 14 departments in nine clinical centers in five different countries (Israel, France, USA, Latvia, and China). 813 patients were diagnosed with one of the following 17 diseases: chronic kidney failure, idiopathic Parkinson's disease, atypical Parkinsonism, multiple sclerosis, Crohn's disease, ulcerative colitis, irritable bowel syndrome, pulmonary arterial hypertension, pre-eclampsia in pregnant women, head and neck cancer, lung cancer, colorectal cancer, bladder cancer, kidney cancer, prostate cancer, gastric cancer, and ovarian cancer. Some of these diseases are not clinically correlated (e.g., preeclampsia and Parkinson's disease), and therefore, they can serve as a model for evaluating the performance of the artificially intelligent nanoarray in disease diagnosis without disturbances of clinically confounding factors. The other diseases do possess clinical correlation between each other (e.g., lung cancer and pulmonary artery hypertension; colorectal cancer and Crohn's disease), and therefore, they can serve as a model for evaluating performance in disease diagnosis with practical clinical interruptions/effects of clinically confounding factors.

An artificially intelligent nanoarray that is based on chemiresistive layers of molecularly modified gold nanoparticles and a random network of single-wall carbon nanotubes was designed and fabricated. The gold nanoparticles were synthesized as previously described [19,20], and dropcasted onto semicircular microelectronic transducers until resistance of several $\mathrm{M} \Omega$ had been reached. The microelectronic transducers consisted of 10 pairs of circular interdigitated (ID) gold electrodes on silicon with $300 \mathrm{~nm}$ thermal oxide. The outer diameter of the circular electrode area was $3 \mathrm{~mm}$, and the gap between two adjacent electrodes and the width of each electrode were both $20 \Omega \mathrm{m}$; the sensors were molecularly modified with different sensing layers, such as dodecanal, 4chlorobenzene methane thiol, and several others.

The SWCNT sensor was based on an electrically continuous random network of SWCNTs formed by drop casting a solution of SWCNTs in dimethylformamide onto the prepared electrical transducers. After deposition, the device was slowly dried overnight under ambient conditions to enhance the self-assembly of the SWCNTs and evaporate the solvent. This procedure was repeated until a resistance of $100 \mathrm{~K} \Omega$ to $10 \mathrm{M} \Omega$ was reached. The microelectronic transducer for the SWCNT sensor consisted of 10 pairs of $4.5 \mathrm{~mm}$ wide interdigitated Ti/Pd electrodes on silicon with $2 \mu$ thermal oxide. The SWCNT sensor was organically functionalized with PAH derivatives. For details regarding each of the sensors, see SI, Table S13. After fabrication, each sensor went through a characterization procedure in which it was exposed to several different concentrations of 
compounds usually found in breath (e.g., isopropyl alcohol, 2-ethyl hexanol, water vapor, and others), in a range of 10 or more parts per billion ( $\mathrm{ppb}$ ) - several part per millions (ppm).

Analysis of the artificially intelligent nanoarray also showed that each disease has its own unique breath print, and that the presence of one disease would not screen out others. Cluster analysis showed a reasonable classification power of diseases from the same categories. The effect of confounding clinical and environmental factors on the performance of the nanoarray did not significantly alter the obtained results.

The diagnosis and classification power of the nanoarray was also validated by an independent analytical technique, i.e., gas chromatography linked with mass spectrometry. This analysis found that 13 exhaled chemical species, called volatile organic compounds, are associated with certain diseases, and the composition of this assembly of volatile organic compounds differs from one disease to another. Overall, these findings could contribute to one of the most important criteria for successful health intervention in the modern era, viz. easy-to-use, inexpensive (affordable), and miniaturized tools that could also be used for personalized screening, diagnosis, and follow-up of a number of diseases, which can clearly be extended by further development.

\section{References}

[1]. V.M. Aroutiounian, Journal of Nanomedicine \& Nanotechnology 11 (2020) 1.

[2]. Ji.W. Yoon, J.H. Lee, Lab on a chip. 1 (2017) 8.

[3]. V.M. Aroutiounian, J. Cont. Phys.-Arm. Acad. Sci. 54 (2009) 324.

[4]. N. Nasiri, Ch. Clark, Biosensors 9 (2019) 43.

[5]. M. Nakhleh, Y. Broza, H. Haick, Nanomedicine 9 (2014) 1991.

[6]. Y.Y. Broza, H. Haick, Nanomedicine 8 (2013) 785.

[7]. R. Vishinkin, H. Haick, Small 11 (2015) 6142.

[8]. G. Konvalina, H. Haick, Acc. Chem. Res. 47 (2014) 66.

[9]. P.I. Gouma, K. Kalyanasundaram, Appl. Phys. Lett. 93 (2008) 244102.

[10]. W. Göpel, Sens. Actuators B 4 (1991) 7.

[11]. N. Shehada, J.C. Cancilla, J.S. Torrecilla et al, ACS Nano 10 (2016) 7047.

[12]. B. Wang, J.C. Cancilla, J.S. Torrecilla et al, Nano Lett. 14 (2014) 933.

[13]. M.K. Nakhleh, S. Baram, R. Jeries et al, Adv. Mater. Technologies 1 (2016) 1600132.

[14]. E. Homede, M. Abo Jabal, R. Ionescu et al, Adv. Funct. Mater. 26 (2016) 6359.

[15]. N. Shehada, G. Brönstrup, K. Funka et al, Nano Lett. 15 (2015) 1288.

[16]. B. Wang, T.-P. Huynh, W. Wu et al, Adv. Mater. 28 (2016), 4012.

[17]. R. Ionescu, Y. Broza, H. Shaltieli et al, ACS Chem. Neurosci. 2 (2011) 687.

[18]. M.K. Nakhleh, H. Amal, R. Jeries et al, ACS Nano 11 (2017) 112.

[19]. G. Peng, U. Tisch, O. Adams et al, Nat. Nanotechnol. 4 (2009) 669.

[20]. E. Dovgolevsky, G. Konvalina, U. Tisch, H. Haick, J. Phys. Chem. C 114 (2010) 14042. 